\title{
Statistical hadronization model description for rapidity densities at RHIC
}

\author{
Jaakko Manninen* \\ Department of Physical Sciences, University of Oulu, Oulu, Finland \\ E-mail: jaakko.manninen@oulu.fi
}

The rapidity densities at $\sqrt{s}_{\mathrm{NN}}=200$ and $130 \mathrm{GeV}$ measured at Relativistic Heavy-Ion Collider by STAR and PHENIX collaborations are analysed within the statistical hadronization model at chemical freeze-out. We find that the model can describe the experimental rapidity densities well. The corresponding chemical freeze-out parameters are determined and they are seen to be in agreement with what we expect from our previous analyses at lower beam energies at AGS and SPS.

The 3rd edition of the International Workshop - The Critical Point and Onset of Deconfinement July 3-7 2006

Galileo Galilei Institute, Florence, Italy

*Speaker. 


\section{Introduction}

We analyze within the statistical hadronization model the rapidity densities at mid-rapidity in the central Au-Au collisions at $\sqrt{s}_{\mathrm{NN}}=200 \mathrm{GeV}$ and $\sqrt{s}_{\mathrm{NN}}=130 \mathrm{GeV}$ measured by STAR and PHENIX collaborations $[1,2,3,4,5,6,7,8,9,10,11,12]$. The analysis is carried out in the grand canonical ensemble with the supplementary $\gamma_{S}$ parameter. This version of the statistical hadronization model is described in detail elsewhere [13, 14].

Statistical model analyses at mid-rapidity at RHIC are often performed by fitting the chemical freeze-out parameters to the ratios of measured rapidity densities $[15,16,17,18,19]$. It is easy to see that in a boost invariant scenario the ratios of rapidity densities represent the ratios of the multiplicities since

$$
\frac{N_{A}}{N_{B}}=\frac{\int\left(d N_{A} / d y\right) d y}{\int\left(d N_{B} / d y\right) d y}=\frac{\left(d N_{A} / d y\right) \int d y}{\left(d N_{B} / d y\right) \int d y}=\frac{d N_{A} / d y}{d N_{B} / d y}
$$

in which the second step results from the fact that the rapidity densities, being boost invariant, are independent on rapidity. We adopt a different approach here and do not include any ratios of rapidity densities in the analysis as this can lead to a large bias in the resulting best fit parameters [20]. Instead, as in our statistical model analyses at AGS and SPS [13, 14], we fit a scaling parameter $V^{\prime}$, common for all particle species, characterizing the size of the hadron emitting source in the limited rapidity window around $y=0$.

We have shown before [13] that performing statistical analysis in a limited rapidity window at SPS energies can artificially enhance the relative strangeness content in the system. This is due to the fact that heavier particles have more narrow rapidity distributions and thus a kinematic cut around mid-rapidity may lead to a situation in which relatively larger fraction of massive particles is taken into account. However, if a sufficiently wide Bjorken type of boost invariant region, much wider than the typical width of the pion rapidity distribution coming from a single cluster at kinetic freeze-out is formed around mid-rapidity, then the rapidity densities of different particle species are approximately constant in this region. In this case it might be possible to determine the characteristics of an average fireball at mid-rapidity without introducing a bias arising from the kinematic cut [14].

\section{Analysis and results}

The rapidity densities in 0-5\% most central Au-Au collisions measured by STAR at $\sqrt{s}_{\mathrm{NN}}=200$ $\mathrm{GeV}$ and statistical hadronization model predictions for the same rapidity densities along our best fit parameters are shown in Table 1. All other rapidity densities are corrected for the weak decay contamination except proton and antiproton rapidity densities which include the weak decay products of $\Lambda$ and $\bar{\Lambda}$. Fit quality is generally good and rapidity densities are well described within the statistical hadronization model. Our findings agree with the similar analysis performed recently by the STAR collaboration [10].

The STAR data in Au-Au collisions at $\sqrt{s}_{\mathrm{NN}}=130 \mathrm{GeV}$ needs to be somewhat manipulated, since some of the rapidity densities are measured in different centrality windows. In order to keep consistency with the STAR 200A GeV data and PHENIX 130A GeV data, we have chosen to 


\begin{tabular}{|c|c|c|}
\hline particle & $\mathrm{dN} / \mathrm{dy}$ & SHM \\
\hline$\pi^{+}[1]$ & $322 \pm 32$ & 325.0 \\
$\pi^{-}[1]$ & $327 \pm 33$ & 327.4 \\
$K^{+}[1]$ & $51.3 \pm 7.7$ & 57.1 \\
$K^{-}[1]$ & $49.5 \pm 7.4$ & 53.5 \\
$p[1]$ & $34.7 \pm 6.2$ & 42.9 \\
$\bar{p}[1]$ & $26.7 \pm 4.0$ & 30.9 \\
$\phi[2]$ & $7.70 \pm 0.30 \pm 0.85$ & 7.10 \\
$\Lambda[10]$ & $16.7 \pm 0.2 \pm 1.1$ & 16.0 \\
$\bar{\Lambda}[10]$ & $12.7 \pm 0.2 \pm 0.9$ & 12.1 \\
$\Xi^{-}[10]$ & $2.17 \pm 0.06 \pm 0.19$ & 1.87 \\
$\bar{\Xi}+[10]$ & $1.83 \pm 0.05 \pm 0.20$ & 1.53 \\
$\Omega+\bar{\Omega}[10]$ & $0.53 \pm 0.04 \pm 0.04$ & 0.63 \\
\hline \hline$V^{\prime} T^{3} e^{-0.7 / T}$ & \multicolumn{2}{|c|}{$12.5 \pm 0.7$} \\
$T[\mathrm{MeV}]$ & \multicolumn{2}{|c|}{$161.0 \pm 3.9$} \\
$\mu_{B}[\mathrm{MeV}]$ & $30.0 \pm 9.8$ \\
$\gamma_{S}$ & \multicolumn{2}{|c|}{$1.02 \pm 0.05$} \\
$\chi^{2} /$ dof & \multicolumn{2}{|c|}{$0.99 \pm 0.04$} \\
$\langle E\rangle /\langle N\rangle[\mathrm{GeV}]$ & \multicolumn{2}{|c}{} \\
\hline
\end{tabular}

Table 1: Top panel: STAR rapidity densities in Au-Au collisions at $\sqrt{s}_{\mathrm{NN}}=200 \mathrm{GeV}$ in the $0-5 \%$ most central collisions. Contribution from weak decays have been subtracted for all particle species except that proton and antiproton rapidity densities include weak decay products of $\Lambda$ and $\bar{\Lambda}$. Errors quoted are statistical + systematic and are taken into account in quadrature. For pions, kaons and nucleons, statistical errors are negligible and thus are not shown. Bottom panel: The statistical hadronization model best fit parameters at chemical freeze-out and the mean energy per particle, averaged over all particle species, determined from the above experimental data.

extrapolate all rapidity densities into the same centrality selection, namely to the $0-5 \%$ most central collisions. This is done by assuming that all final hadronic rapidity densities scale linearly

$$
\frac{d N}{d y}=a+b \frac{d N_{h^{-}}}{d \eta}
$$

with the negative hadron pseudorapidity density $\mathrm{d} N_{h^{-}} / \mathrm{d} \eta$ in central and semi-central collisions. This is approximately true in the boost invariant scenario around mid-rapidity provided that soft processes dominate the particle production and all particle multiplicities are proportional to the negative hadron multiplicity. This seems to hold true relatively well for all the measured particle species.

Using the STAR inelastic cross section versus $\mathrm{d} N_{h^{-}} / \mathrm{d} \eta$ data from [3], we have calculated the negative hadron pseudorapidity densities $\mathrm{d} N_{h^{-}} / \mathrm{d} \eta$ in several centrality windows in the central pseudorapidity region $(|\eta|<0.5)$ in order to estimate the rapidity densities for each of the particle species in the most central collision window. Particularly, we have calculated $\mathrm{d} N_{h^{-}} / \mathrm{d} \eta=297$ in the $0-5 \%$ most central collisions. This is in good agreement with STAR collaboration estimate 
$\mathrm{d} N_{h^{-}} / \mathrm{d} \eta \approx 300 \pm 6 \%[8,4]$ and we have chosen the same $6 \%$ as our error leading to the $\mathrm{d} N_{h^{-}} / \mathrm{d} \eta=$ $297 \pm 18$ in the $0-5 \%$ most central collisions.

Pions and lambdas are already measured in the $0-5 \%$ most central collisions and need no manipulation while the rapidity densities for other particle species need to be extrapolated. The negative hadron pseudorapidity densities as a function of centrality are published along kaon rapidity densities [5] in various centrality windows and thus we have fitted Eq. (2.1) to the data and used $\mathrm{d} N_{h^{-}} / \mathrm{d} \eta=297 \pm 18$ to estimate the $K^{ \pm}$rapidity densities in the $0-5 \%$ most central collisions. Protons and antiprotons [7] are measured in the same centrality windows as kaons and thus we have used the same negative hadron pseudorapidity densities as for kaons in order to estimate the $p$ and $\bar{p}$ rapidity densities in the $0-5 \%$ most central collisions.

$\Xi^{-}$and $\Xi^{+}[9]$ as well as $\phi[6]$ are measured in three different centrality windows and similarly to kaons and nucleons, we have estimated rapidity densities in the $0-5 \%$ most central collisions by assuming linear dependence on the negative hadron pseudorapidity density. Unlike in the case of kaons and nucleons, the negative hadron pseudorapidity densities estimated by STAR collaboration in these windows deviate somewhat from the ones we have calculated. In order to keep the same overall scale, we have used our calculated pseudorapidity spectrum to estimate the $\Xi^{ \pm}$and $\phi$ rapidity densities in the $0-5 \%$ most central collisions. Also in [9], the $0-10 \%$ most central rapidity density for $\Omega+\bar{\Omega}$ is given and without better knowledge, we have estimated the $\Omega+\bar{\Omega}$ rapidity density in the $0-5 \%$ most central collisions by scaling the yields with a factor $\left(d N_{h^{-}} / d \eta\right)_{5 \%} /\left(d N_{h^{-}} / d \eta\right)_{10 \%}=1.06$. No weak decay corrections have been applied to any of the rapidity densities except $\pi^{ \pm}$densities do not include weak decay products of $\Lambda, \bar{\Lambda}$ and $K_{s}^{0}$.

Table 2 shows the extrapolated (experimental) and the statistical hadronization model predictions for the rapidity densities in the $0-5 \%$ most central collisions as well as the statistical model best fit parameters in Au-Au collisions at $\sqrt{s}_{\mathrm{NN}}=130 \mathrm{GeV}$. Again, the fit quality is good and most of the rapidity densities are described well within the statistical hadronization model. The chemical freeze-out temperature is about the same and baryon chemical potential is few $\mathrm{MeV}$ higher than in central Au-Au collisions at $200 \mathrm{~A} \mathrm{GeV}$. Unexpectedly the strangeness under-saturation parameter $\gamma_{S}$ seems to be significantly over unity. To cross check our results, we can analyze the rapidity densities in central Au-Au collisions at the same center-of-mass energy $\sqrt{s}_{\mathrm{NN}}=130 \mathrm{GeV}$ measured by the PHENIX collaboration. PHENIX has measured a subset of the particle species measured by STAR, namely $\pi^{ \pm}, K^{ \pm}, p, \bar{p}, \Lambda$ and $\bar{\Lambda}[12,11]$. Our extrapolated rapidity densities agree with the corresponding PHENIX values (see Tables 2 and 3). No weak decay corrections have been applied to the PHENIX data which is the reason why $\pi^{ \pm}$rapidity densities deviate among PHENIX and STAR data at the same beam energy. In all cases, strong and weak decays corresponding to the experimental definitions are taken carefully into account in our analyses. The statistical model best fit parameters estimated from the PHENIX data agree with the ones coming from a fit to the larger data sample measured by STAR. This rules out the possibility that our extrapolations with STAR data would bias the fit towards unexpectedly large $\gamma_{S}$.

We have repeated the analysis with $\gamma_{S}$ fixed to unity both with the STAR and PHENIX rapidity densities at $\sqrt{s}_{\mathrm{NN}}=130 \mathrm{GeV}$. It seems that the minimum of the $\chi^{2}$ distribution is relatively flat in the case of the PHENIX data and the rapidity densities can be described well $\left(\chi^{2} / d o f=2.0 / 3\right)$ with the statistical hadronization model in absolute chemical equilibrium as well. The other resulting chemical freeze-out parameters are virtually the same as in Table 3. Setting $\gamma_{S}=1$ with the STAR ra- 


\begin{tabular}{|c|c|c|}
\hline particle & $\mathrm{dN} / \mathrm{dy}$ & SHM \\
\hline$\pi^{+}[8]$ & $239.0 \pm 3.0 \pm 2.0 \pm 10.0$ & 233.8 \\
$\pi^{-}[8]$ & $239.0 \pm 3.0 \pm 2.0 \pm 10.0$ & 236.6 \\
$\Lambda[4]$ & $17.20 \pm 0.4 \pm 1.72$ & 17.98 \\
$\bar{\Lambda}[4]$ & $12.3 \pm 0.3 \pm 1.23$ & 12.5 \\
\hline$K^{+}$ & $45.75 \pm 0.6 \pm 6.0 \pm 2.85$ & 46.92 \\
$K^{-}$ & $43.16 \pm 0.6 \pm 5.4 \pm 2.57$ & 43.36 \\
$p$ & $26.15 \pm 0.23 \pm 5.8 \pm 1.63$ & 32.17 \\
$\bar{p}$ & $18.85 \pm 0.16 \pm 4.12 \pm 1.15$ & 21.96 \\
$\Xi^{-}$ & $2.13 \pm 0.14 \pm 0.20 \pm 0.11$ & 1.80 \\
$\bar{\Xi}^{+}$ & $1.78 \pm 0.12 \pm 0.17 \pm 0.12$ & 1.42 \\
$\Omega+\bar{\Omega}$ & $0.586 \pm 0.11 \pm 0.056 \pm 0.035$ & 0.702 \\
$\phi$ & $6.09 \pm 0.37 \pm 0.69 \pm 0.34$ & 6.75 \\
\hline \hline$V^{\prime} T^{3} e^{-0.7 / T}$ & \multicolumn{2}{|c|}{$8.46 \pm 0.45$} \\
$T[\mathrm{MeV}]$ & $160.5 \pm 4.3$ \\
$\mu_{B}[\mathrm{MeV}]$ & $35.6 \pm 12.8$ \\
$\gamma_{S}$ & $1.20 \pm 0.08$ \\
$\chi^{2} / d o f$ & $7.0 / 8$ \\
$\langle E\rangle /\langle N\rangle[\mathrm{GeV}]$ & $1.00 \pm 0.04$ \\
\hline
\end{tabular}

Table 2: Top panel: Estimated rapidity densities in Au-Au collisions at $\sqrt{s}_{\mathrm{NN}}=130 \mathrm{GeV}$ in the $0-5 \%$ most central collisions measured by STAR collaboration. Rapidity densities of pions and lambdas are measurements while rapidity densities of other particle species are our estimates. Errors for our estimates are statistical + systematic + our extrapolation and are taken into account in quadrature. Statistical and systematic errors quoted are the experimental errors in the most central experimentally accessible window, which are $0-6 \%$ for $K^{ \pm}, p$ and ${ }^{-} p, 0-10 \%$ for ${ }^{ \pm}$and $\Omega+\bar{\Omega}$ and $0-11 \%$ for $\phi$. No weak decay corrections have been applied except $\pi^{ \pm}$rapidity densities do not include weak decay products of $\Lambda, \bar{\Lambda}$ and $K_{s}^{0}$. Bottom panel: The statistical hadronization model best fit parameters at chemical freeze-out and the mean energy per particle determined from the above experimental data.

pidity densities leads to somewhat larger temperature $T=169.1 \pm 4.4 \mathrm{MeV}$ while baryon chemical potential is essentially unaffected. The anomalous behavior of strangeness phase-space occupancy factor at $\sqrt{s}_{\mathrm{NN}}=130 \mathrm{GeV}$ is not yet well understood and the issue deserves further consideration.

\section{Discussion and Conclusions}

The resulting chemical freeze-out parameters can be compared with the ones we have determined in central heavy-ion collisions at lower beam energies at AGS and SPS [14]. One should notice that the previous analyses have been performed with full phase-space multiplicities while the statistical hadronization model analysis at RHIC employs rapidity densities measured in a limited rapidity window around mid-rapidity. Nevertheless, statistical hadronization model parameters in central Au-Au collisions at RHIC seem to be in good agreement with our interpolating curves for statistical hadronization model parameters determined at lower beam energies, see Figure 1. The 


\begin{tabular}{|c|c|c|}
\hline particle & $\mathrm{dN} / \mathrm{dy}$ & SHM \\
\hline$\pi^{+}$ & $276 \pm 3 \pm 36$ & 264.4 \\
$\pi^{-}$ & $270 \pm 3.5 \pm 35$ & 269.6 \\
$K^{+}$ & $46.7 \pm 1.5 \pm 7.0$ & 46.2 \\
$K^{-}$ & $40.5 \pm 2.3 \pm 6.1$ & 42.9 \\
$p$ & $28.7 \pm 0.9 \pm 4.0$ & 29.6 \\
$\bar{p}$ & $20.1 \pm 1.0 \pm 2.8$ & 20.6 \\
$\Lambda$ & $17.3 \pm 1.8 \pm 2.8$ & 15.9 \\
$\bar{\Lambda}$ & $12.7 \pm 1.8 \pm 2.0$ & 11.8 \\
\hline \hline$V^{\prime} T^{3} e^{-0.7 / T}$ & \multicolumn{2}{|c|}{$8.07 \pm 0.11$} \\
$T[\mathrm{MeV}]$ & $158.0 \pm 5.9$ \\
$\mu_{B}[\mathrm{MeV}]$ & $33.5 \pm 17.8$ \\
$\gamma_{S}$ & $1.24 \pm 0.22$ \\
$\chi^{2} / d o f$ & $0.5 / 4$ \\
$\langle E\rangle /\langle N\rangle[\mathrm{GeV}]$ & $0.97 \pm 0.06$ \\
\hline
\end{tabular}

Table 3: Top panel: Rapidity densities around $|y|=0$ in central Au-Au collisions at $\sqrt{s}_{\mathrm{NN}}=130 \mathrm{GeV}$ measured by the PHENIX collaboration. All data taken from [12] except lambdas from [11]. No weak decay corrections have been applied to any of the particle species. Errors quoted are statistical + systematic and are taken into account in quadrature in the analysis. Bottom panel: The statistical hadronization model best fit parameters at chemical freeze-out and the mean energy per particle determined from the above experimental data.

solid line indicates the beam energy region in which the interpolating curves are determined while the dashed part is an extrapolation to RHIC energies. For the explicit formulas and details, see [14].

Figure 2 shows the strangeness phase-space occupancy factor $\gamma_{S}$ as a function of the center-ofmass energy of a colliding nucleon pair in central heavy-ion collisions. One can see that strangeness seems to reach absolute chemical equilibrium in central heavy-ion collisions at RHIC energies only. From the right panel of Figure 2 one can see that the RHIC systems at 200 and $130 \mathrm{~A} \mathrm{GeV}$ seem to follow the chemical freeze-out curve $\langle E\rangle /\langle N\rangle=1 \mathrm{GeV}$ [21].

\section{References}

[1] J. Adams et al. [STAR Collaboration], Phys. Rev. Lett. 92 (2004) 112301.

[2] J. Adams et al. [STAR Collaboration], Phys. Lett. B 612 (2005) 181.

[3] C. Adler et al. [STAR Collaboration], Phys. Rev. Lett. 87 (2001) 112303.

[4] C. Adler et al. [STAR Collaboration], Phys. Rev. Lett. 89 (2002) 092301.

[5] C. Adler et al. [STAR Collaboration], Phys. Lett. B 595 (2004) 143.

[6] C. Adler et al. [STAR Collaboration], Phys. Rev. C 65 (2002) 041901.

[7] J. Adams et al. [STAR Collaboration], Phys. Rev. C 70 (2004) 041901.

[8] J. Adams et al. [STAR Collaboration], arXiv:nucl-ex/0311017. 

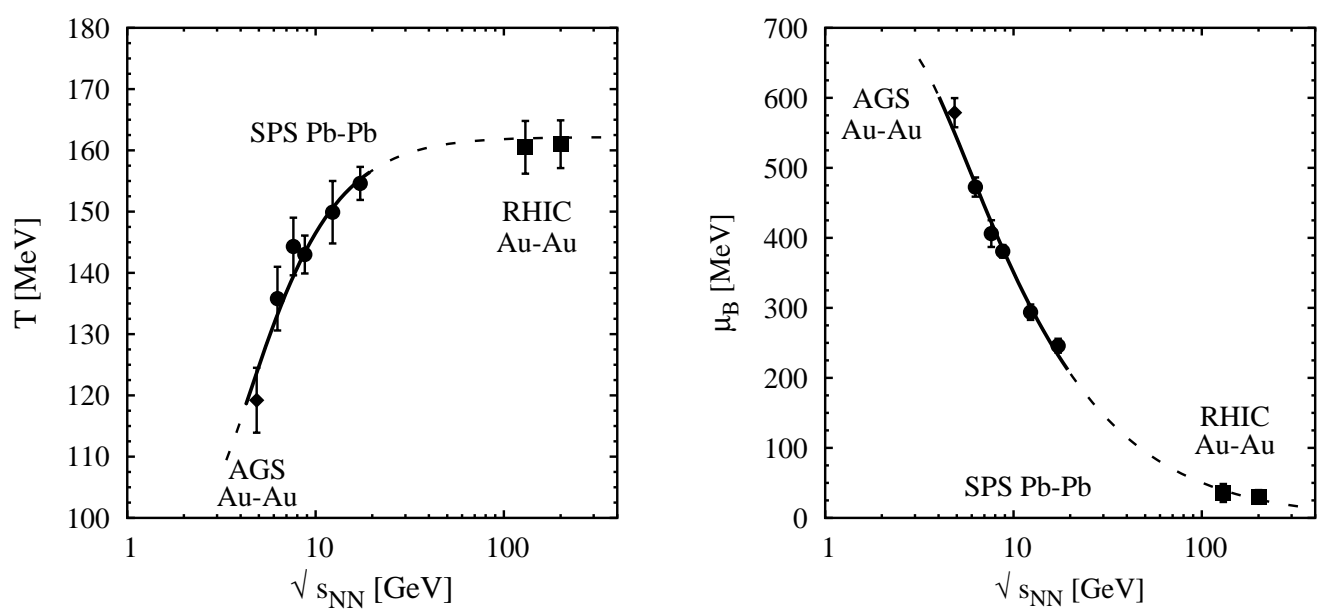

Figure 1: Chemical freeze-out temperature (LEFT) and baryon chemical potential (RIGHT) as a function of the center-of-mass energy of a colliding nucleon pair in various central collision systems. The lines are our interpolating curves for central heavy-ion collisions [14].
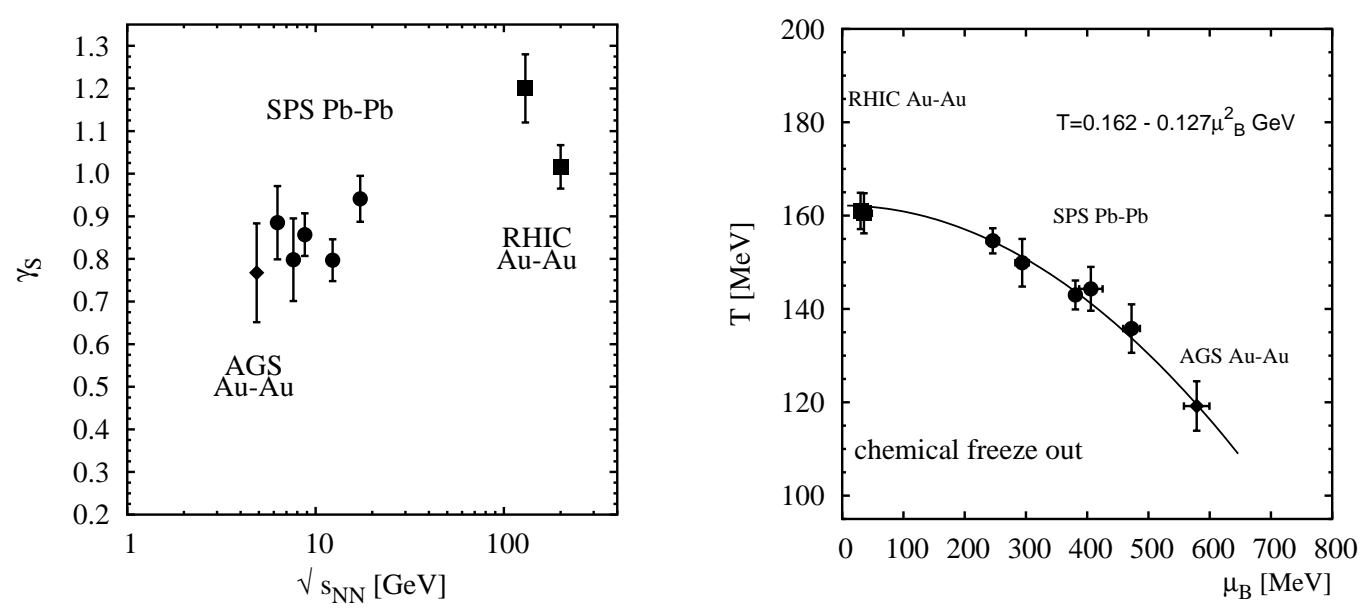

Figure 2: LEFT: Strangeness under-saturation parameter $\gamma_{S}$ as a function of the center-of-mass energy of a colliding nucleon pair in various central collision systems.

RIGHT: The chemical freeze-out curve in central heavy-ion collisions. The line represents the condition $\langle E\rangle /\langle N\rangle=1 \mathrm{GeV}$.

[9] J. Adams et al. [STAR Collaboration], Phys. Rev. Lett. 92 (2004) 182301.

[10] J. Adams et al. [STAR Collaboration], arXiv:nucl-ex/0606014.

[11] K. Adcox et al. [PHENIX Collaboration], Phys. Rev. Lett. 89 (2002) 092302.

[12] K. Adcox et al. [PHENIX Collaboration], Phys. Rev. C 69 (2004) 024904.

[13] F. Becattini, M. Gazdzicki, A. Keranen, J. Manninen and R. Stock, Phys. Rev. C 69 (2004) 024905.

[14] F. Becattini, J. Manninen and M. Gazdzicki, Phys. Rev. C 73 (2006) 044905.

[15] P. Braun-Munzinger, D. Magestro, K. Redlich and J. Stachel, Phys. Lett. B 518 (2001) 41. 
[16] A. Baran, W. Broniowski and W. Florkowski, Acta Phys. Polon. B 35 (2004) 779.

[17] J. Cleymans, B. Kampfer, M. Kaneta, S. Wheaton and N. Xu, Phys. Rev. C 71, 054901 (2005).

[18] A. Dumitru, L. Portugal and D. Zschiesche, Phys. Rev. C 73 (2006) 024902.

[19] A. Andronic, P. Braun-Munzinger and J. Stachel, Nucl. Phys. A 772 (2006) 167.

[20] F. Becattini, These proceedings.

[21] J. Cleymans and K. Redlich, Phys. Rev. Lett. 81 (1998) 5284. 